\title{
Taylor's Formula, Limited Development, and Development of Power Series: A Study of the Knowledge of University Professors in Training
}

\author{
Edgardo Locia-Espinoza ${ }^{1 *}$, Armando Morales-Carballo ${ }^{1}$, Héctor Merino-Cruz ${ }^{1}$ \\ ${ }^{7}$ Autonomous University of Guerrero, MEXICO \\ *CORRESPONDENCE: \lociae999@hotmail.com
}

\begin{abstract}
This paper reports the results of three questionnaires applied to sixty-seven students preparing to become university-level mathematics teachers; the questionnaires were focused on knowing their conceptions and their mastery of the representations of functions in the development of power series. The theoretical and methodological background rests on the Mathematics Teacher's Specialised Knowledge (MTSK), specifically in the domain: Mathematical Knowledge (MK).

As a result of the analysis of the responses to the questionnaires, it was identified that the notion of development of power series played an important role as a means of justification and that from the three notions addressed (Taylor's Formula, limited development, development of power series), this was the most prevalent in the mind of the students. These results will be used as the starting point for the development of proposals that improve the teaching and learning of the subject of study.
\end{abstract}

Keywords: Taylor's Formula, development of power series, the teaching of calculus and analysis

\section{INTRODUCTION}

The teacher's activity in the teaching-learning process is major, since the teacher accompanies and directly influences the student's learning. The basic exigency on the teacher is related to the solid mastery of the mathematical content of the subject of teaching and with the methodological preparation required for the development and implementation of teaching-learning activities.

From the authors' point of view, teaching and learning are two linked processes. One cannot speak of learning if there is no good preparation of the teaching activity; and vice versa, the teaching activity is also a function of the needs raised as learning objectives. Therefore, in the teaching-learning process, the fundamental elements are: the teacher, the student and knowledge.

For a long time, research in the field of mathematics education was directed towards the student or the curriculum. Recently, the research trends are directed to the teacher too. In this respect, some theoretical models have been developed to carry out studies relating to their conceptions, the mastery of mathematical content and teaching methodologies.

On the other hand, there are two critical situations in the study of the teaching and learning processes of mathematics at different educational levels; the first one is linked with the comprehension of mathematics from its concepts, notions, theorems, and knowledge. The second one is linked to the domain of the procedures, calculations, and algorithms. However, during the teaching practice, we are aware that many students know

Article History: Received 30 January $2020 \bullet$ Revised 5 March $2020 \bullet$ Accepted 8 March 2020

(C) 2020 by the authors; licensee Modestum Ltd., UK. Open Access terms of the Creative Commons Attribution 4.0 International License (http://creativecommons.org/licenses/by/4.0/) apply. The license permits unrestricted use, distribution, and reproduction in any medium, on the condition that users give exact credit to the original author(s) and the source, provide a link to the Creative Commons license, and indicate if they made any changes. 
how to apply the algorithms and do the calculations correctly but forget the meaning of the definitions of the mathematical objects. Some investigations suggest that even students who are trained in mathematics "sympathize" more with calculations and prefer to work algorithmically over more theoretical exercises (Antibi, 1988). This constitutes a problem because many students find little interest in content and prefer to apply an algorithm to obtain a result quickly, without using axioms, theorems, definitions, and concepts, among others (Abarca, 2007).

Another issue associated with these situations is the one that refers to the symbols and their meanings. Students often learn symbols by heart and forget their meanings. In some cases, these situations arise because the substantial problems (linked to the content) are frequently solved with formal procedures. This procedure means that the algorithms and formalizations are introduced without ensuring a sufficient understanding of the content. According to Polya (1958), "methods will not be understood if they are taught without their motives; rules will be quickly forgotten if they are given without explanations or relationships between them. A course will be transformed into a kind of disjointed stock with no possibility to provide the least manipulative information if all the forms of reasoning are excluded." Mathematical objects are frequently introduced without considering the development of the right images of the content of the object. This is substituted with the introduction of the corresponding names and symbols. It is necessary to develop these images and then take care of the preservation of the relationships between the symbols and their representations in order to avoid the production or accumulation of purely formal knowledge in the mind of the students.

Usually, students forget the studied notions over time, especially if they do not use them during an extended period. However, it is not a complete oblivion. Some of these notions remain in their mind, and it is not difficult to assume that they are those that are the most meaningful for them. It is also common to assume that the basic concepts are easily forgotten, in terms of meaning, when the education received by the students is abundant with "recipes." In this regard, we could ask the following questions: To what extent are representations formalized? About the given content, how familiar should students be with formalizations?

Some difficulties in understanding concepts related to Mathematical Analysis (Real and Complex) have been identified according to the experience of the authors in the training and professionalization of mathematics teachers for pre-university and university levels.

In the period between February and July 2019, ten in-service mathematics teachers and students from the Advanced Calculus II undergraduate course of the Mathematics Educational degree at the Autonomous University of Guerrero were interviewed in order to ask about their abilities to handle the concepts, procedures, and applications of Differential and Integral Calculus and Mathematical Analysis. In particular, they were asked about optimization problems and the development of a power series. In this regard, it was identified that eight of the ten teachers have only superficial ideas about the methods used to solve these types of problems, and only mentioned the geometrical series concerning the power series and were not able to explain their behavior.

Different tests on optimization problems and Taylor's Formula (contents of the courses of Differential Calculus of one and several variables), on the use of power series in the study of differential equations, and the treatment of complex variable taken by students of the Mathematics Education degree course at the Autonomous University of Guerrero allowed us to identify that they were able to manipulate the geometric series and develop the power series using the Taylor-Maclaurin formula, but have great difficulty considering the conditions that guarantee its convergence.

In particular, little has been explored, at least on a local level, about the students' mastery of the procedures that refer to the Limited development (LD), Taylor's Formula (TF), and the Development of Power Series (DPS). However, this exploration is crucial; collecting this information will be the starting point for the development of proposals for teaching these contents of Calculus and Mathematical Analysis the university level.

The study of these concepts (LD, TF, and DPS) is crucial because its applications favor the study of the local behavior of a function and its polynomial approximation, the estimation of irrational numbers bounding errors, and the estimation of integrals among others, essentially in the field of Mathematics Analysis.

With the aim to identify the mathematical knowledge of the students about these concepts (LD, TF and DPS) and being able to support the development of a proposal for teaching and learning these topics, we set the following objective in this work: Analyze the reactions of the students in the presence of a function that admits the three types of representations: TF, LD, and DPS; it is of particular interest to identify which of these representations is the most prevalent in the mind of the students and if they have in mind the conditions 
that a function must satisfy so that it can be developed at the time of solving an LD problem or if their work is purely algorithmic.

\section{THEORETICAL FOUNDATION}

The line of research in teacher education has been of great interest for several investigators from different theoretical perspectives (e. g. Carrillo et al. 2014; Godino, 2009; Ball, Thames \& Phelps, 2008; Shulman, 1986, among others). One of the main concerns in this line of research is the study of the knowledge of the mathematics teachers to analyze its nature, characteristic, degree of actual or required knowledge to develop their teaching assignments (Rojas, Flores \& Carrillo, 2015). The importance of studying the professional knowledge of mathematics teachers lies in the fact that it is the teacher who guides the process of learning mathematics, implements the learning activities developed according to the mastery of the mathematical content, the orientation established in the curricula, and the methodological resources available.

The works of Shulman $(1986,1987)$ have provided a basis for a range of theoretical proposals to understand and characterize the professional practices of mathematics teachers which include the Model of Mathematical Knowledge for Teaching (MKT) developed by Ball, Thames and Phelps (2008), and the model of Mathematics Teacher's Specialised Knowledge (MTSK) of Carrillo et al. (2014). The MKT model consists of two domains of knowledge: the subject matter knowledge and the pedagogical content knowledge, and the MTSK model that considers, as the previous model, the mathematical knowledge and the pedagogical content knowledge.

\section{Model of Mathematical Knowledge for Teaching}

The model of mathematical knowledge for teaching of Shulman (1986) considers three categories for content knowledge: (a) the content knowledge of the subject, which refers to the amount and mental organization that a teacher has about the content he will teach; for example, Shulman considers in this subdomain that teachers must not only be able to define the accepted truths in a domain to their students, they must also be able to explain why a specific proposition is justified, why it is worthy of knowing it and how it is related to other propositions both within and outside of the discipline, and both in theory and practice; (b) pedagogical content knowledge, which transcends the subject matter and deals with the knowledge of how to teach it; this category includes the most useful forms of representation, analogies, illustrations, examples, explanations, and the essential demonstrations so that the subject is more comprehensible to students; and (c) the curriculum knowledge, which consists of a thorough knowledge of the curriculum. According to Shulman, the curriculum is represented by the programs designed for teaching specific topics at a given level, the variety of materials, information available related to these programs, the set of characteristics that serve as indications and contraindications for the use of a particular curriculum or program materials in particular circumstances.

\section{Model of Mathematics Teacher's Specialised Knowledge}

The MTSK model considers two sub-domains of knowledge: mathematical knowledge (MK), and the pedagogical content knowledge (PCK), which, in turn, are composed of different sub-domains. This model also considers the teacher's beliefs or conceptions about mathematics, teaching, and learning processes. We will now show each of the sub-domains described by Carrillo et al. (2014):

The mathematical knowledge is composed of the following sub-domains:

- Knowledge of mathematical topics refers to the fact that the mathematics teachers should know the mathematical contents and their meanings in an informed way; that is, concepts, procedures, records of representation, properties. This sub-domain integrates the content that students must learn and allows the consideration of more in-depth knowledge by the teacher than the one expected by the students.

- Knowledge of the structure of mathematics, it is the knowledge of the relationships that a teacher makes between different contents, either from the course he is teaching or from other courses or educational levels. It is specifically about connections between mathematical topics.

- Knowledge about mathematical practice, this sub-domain highlights the importance that the teacher not only knows the established mathematical results but also the ways to proceed to reach them and the characteristics of mathematical work. It is about knowing how knowledge is explored and generated in mathematics, how relationships, correspondences, and equivalencies are established, how it is 
argued, it is reasoned and generalized, the role that agreement has, and the characteristics that have some of the elements with which mathematics is made.

The pedagogical content of knowledge is formed by the following sub-domains:

- Knowledge of features of learning mathematics. This sub-domain encompasses knowledge about the learning characteristics inherent to the mathematical content. It avoids looking at the student as the main focus of the process by shifting the look towards the mathematical content as a learning object. It is interested in knowledge related to the characteristics of learning derived from the interaction between the mathematical content and not with the specific characteristics of the student.

- Knowledge of teaching mathematics. This sub-domain includes the knowledge of resources, materials, ways of presenting contents, and their potential to be used for teaching, as well as the knowledge of proper examples for each content, intention, or context.

- Knowledge of mathematics learning standards. This sub-domain considers the knowledge that a teacher has about the specifications of what a student must learn and the conceptual level that is expected in a specific curricular moment.

The common consideration between all of these models is that the teachers should master the corresponding disciplinary contents, even when it is common to accept that it is not enough to guarantee the professional competence (Godino, 2009). However, we consider that disciplinary knowledge is really necessary for teaching because it is not possible to teach what is not known. The MTSK model "does not intend to assess whether the teacher knowledge is correct or incorrect, but tries to show a thorough description of it" (EscuderoÁvila, 2015, p.65), this means that MTSK does not give any value judgment, but rather intends to understand (through categories of analysis and indicators of knowledge) the knowledge that the teacher has for his teaching.

From this perspective, this paper uses the fundamental components of the MTSK model focused on mathematical knowledge. Specifically, this background is used to explain the knowledge that trainee teachers have about Taylor's Formula, Limited Development, and Development of Power Series, and their differences and characterization.

\section{Definitions and Important Results}

\section{Taylor's Formula (TF)}

This formula is considered immediately after working with finite increments. It is established as an existence theorem, which means that under specific conditions that a function $f$ and its derivatives must satisfy in an interval (f must be of class $C^{n}$ on $[a, b]$ and $n+1$ times differentiable), the existence of an expression of the form $f(b)$ is always guaranteed:

$$
f(b)=f(a)+\frac{f^{\prime}(a)}{1 !}(b-a)+\frac{f^{\prime \prime}(a)}{2 !}(b-a)^{2}+\cdots+\frac{f^{(n)}(a)}{n !}(b-a)^{n}+\frac{f^{(n+1)}(c)}{(n+1) !}(b-a)^{n+1},
$$

where $c$ is a point that belongs to the open interval $(a, b)$.

If these conditions are satisfied on an interval that contains 0 , and if $x$ belongs to this interval, then we can lead to the expression

$$
f(x)=f(0)+\frac{f^{\prime(0)}}{1 !} x+\frac{f^{\prime \prime(0)}}{2 !} x^{2}+\cdots+\frac{f^{(n)}(0)}{n !} x^{n}+\frac{f^{(n+1)}(c)}{(n+1) !} x^{n+1}, \text { where }|c|<|x| .
$$

The former expression is called Taylor-McLaurin's Formula with Lagrange's Remainder. The polynomial

$$
f(0)+\frac{f^{\prime}(0)}{1 !} x+\frac{f^{\prime \prime}(0)}{2 !} x^{2}+\cdots+\frac{f^{(n)}(0)}{n !} x^{n},
$$

is called the regular part, and the last term is called Lagrange's Remainder. This is, $f(x)=P(x)+R(x)$, where $P(x)=$ regular part and $R(x)=$ Lagrange's Remainder. We should point out that there are other forms of the remainder of Taylor's Formula.

\section{Limited Development (LD)}

These developments are frequently used in the calculations of limits. The introduction to this notion is made through a definition. Let $f$ be a function defined in a neighborhood of $a$, except perhaps for $x=a$. It is said that $\mathrm{f}$ admits a limited development of order $n$, in a neighborhood of $x=a$, if it can be written as 


$$
f(x)=a_{0}+a_{1}(x-a)+a_{2}(x-a)^{2}+\cdots+a_{n}(x-a)^{n}+(x-a)^{n} \varepsilon(x), \text { where } \lim _{x \rightarrow a} \varepsilon(x)=0 .
$$

If $a=0$, then there is a limited distribution of order $n$ in zero

$$
f(x)=a_{0}+a_{1} x+a_{2} x^{2} \ldots+a_{n} x^{n}+x^{n} \varepsilon(x) \text {, donde } \lim _{x \rightarrow 0} \varepsilon(x)=0 .
$$

It is important to highlight the fact that a function does not necessarily have an LD of order $\mathrm{n}$, but if it exists, then it is unique. The first relationship that can be established between the limited developments and Taylor's formula is the theorem of Taylor-Young.

Theorem 1 (of Taylor-Young): If $f$ is class $C^{n-1}$ in a neighborhood of $a$ and if $f^{(n)}(a)$ exists, then the function $f$ has a limited expansion in a neighborhood of $a$, of order $n$. This limited development is given by the formula of Taylor-Young

$$
f(x)=f(a)+\frac{f^{\prime}(a)}{1 !}(x-a)+\frac{f^{\prime \prime}(a)}{2 !}(x-a)^{2}+\cdots+\frac{f^{(n)}(a)}{n !}(x-a)^{n}+(x-a)^{n} \varepsilon(x),
$$

where $\lim _{x \rightarrow a} \varepsilon(x)=0$.

The idea for the demonstration of this theorem lies in proving that under these conditions, the factor $\left|f^{(n)}(c)\right|$, that appears in Lagrange's Remainder, is bounded ( $c$ belongs to a neighborhood of $a$ ).

This result will give us the first LD's and many others will be deduced from them with the aid of theorems related to the operations (addition, product, and quotient) and compound functions, derivation, and integration. These theorems will be called the Classic Theorems of the limited developments in this work.

\section{Development of Power Series (DPS)}

It is defined in the following way: It is said that $f$ can be expressed in a power series in a neighborhood of $a$, if there is a power series $\sum a_{n}(x-a)^{n}$ that has a nonzero radius of convergence $r$ such that

$$
f(x)=a_{0}+a_{1}(x-a)+a_{2}(x-a)^{2}+\cdots
$$

Relationship between the three notions. The relationships between the three developments are established by the following theorems.

Theorem 2: If $f$ is a function of class $C^{\infty}$ in a neighborhood of 0 , then:

a) $f$ admits a Taylor development of any order in a neighborhood of 0 .

$$
f(x)=f(0)+\frac{f(0)}{1 !} x+\frac{f^{\prime \prime}(0)}{2 !} x^{2}+\cdots+\frac{f^{(n)}(0)}{n !} x^{n}+\frac{f^{(n+1)}(c)}{(n+1) !} x^{n+1}
$$

where $|c|<|x|$.

b) $f$ admits a limited development of any order in 0 .

Theorem 3: If $f$ admits a DPS in a neighborhood of 0 , then $f$ is of $C^{\infty}$ in a neighborhood of 0 .

Observation: A $C^{\infty}$ function in a neighborhood of 0 , does not necessarily admit a DPS in 0 .

To prove this, it is enough to consider the function $f$ defined as

$$
f(x)=e^{-1 / x^{2}} \text {, si } x \neq 0 \text { y } f(0)=0 .
$$

Expressions of the remainder. The "last terms" of the three previous representations are shown below

$\mathrm{TF}: f(x)=f(0)+\frac{f(0)}{1 !} x+\frac{f^{\prime \prime}(0)}{2 !} x^{2}+\cdots+\frac{f^{(n)}(0)}{n !} x^{n}+\frac{f^{(n+1)}(c)}{(n+1) !} x^{n+1}$

LD: $f(x)=a_{0}+a_{1} x+a_{2} x^{2} \ldots+a_{n} x^{n}+x^{n} \varepsilon(x)$, donde $\lim _{x \rightarrow 0} \varepsilon(x)=0$

DPS: $f(x)=a_{0}+a_{1} x+a_{2} x^{2} \ldots+a_{n} x^{n}+\cdots$

The primary focus are the expressions indicated in Table 1.

Table 1. Last terms of TF, LD and DPS

\begin{tabular}{ccc}
\hline TF & LD & DPS \\
\hline$\frac{f^{(n+1)}(c)}{(n+1) !} x^{n+1}$ & $x^{n} \varepsilon(x)$ & “..” \\
\hline
\end{tabular}

We know that there are relationships between the three notions. Therefore, we raised the following questions: Do students understand the meaning of each of these expressions of the remainders? What meaning 
do students give to the ellipsis in the expression of the development of power series? About $\frac{f^{(n+1)}(c)}{(n+1) !} x^{n+1}$, do they understand that point $\mathrm{c}$ depends on point $x$ ? Is the expression " $x{ }^{n} \varepsilon(x)$, where $\lim _{x \rightarrow 0} \varepsilon(x)=0$ " only a symbol in which the exponent of $x$ must be the same as the exponent of $x$ in the last term of the regular part? Which of the three notions is the "most natural" for most of the students?

\section{METHOD}

\section{Preparation and Validation of the Questionnaires}

The system of activities that were distributed in the three questionnaires was validated using the criterion of experts. This criterion, as an empirical method in mathematics education, is based on the consultation with people with thorough knowledge and educational experience on the topic that is investigated or experienced in the context in which it develops, and the goal is to collect first-hand criteria on the object of investigation.

\section{Selection and Number of Experts}

Five experts were consulted. The selection criteria used was as follows: The experts have extensive knowledge in research, Methodology of Teaching Mathematics, and Mathematics. Table 2 shows the information of the experts consulted for the validation of the system of activities grouped in the three questionnaires.

Table 2. Information from the experts consulted

\begin{tabular}{|c|c|c|}
\hline Expert Scientific degree & Research professor & Domains \\
\hline $\mathrm{PhD}$ & Yes & $\begin{array}{l}\text { Calculus and Mathematical Analysis. } \\
\text { Research in the didactics of Mathematics. } \\
\text { Methodology in Mathematics Education. }\end{array}$ \\
\hline $\mathrm{PhD}$ & Yes & $\begin{array}{l}\text { Calculus and Mathematical Analysis. } \\
\text { Research. }\end{array}$ \\
\hline $\mathrm{PhD}$ & Yes & $\begin{array}{l}\text { Calculus and Mathematical Analysis. } \\
\text { Research. }\end{array}$ \\
\hline $\mathrm{PhD}$ & Yes & $\begin{array}{l}\text { Calculus and Mathematical Analysis. } \\
\text { Research in the didactics of Mathematics. } \\
\text { Methodology in Mathematics Education. }\end{array}$ \\
\hline $\mathrm{PhD}$ & Yes & $\begin{array}{l}\text { Calculus and Mathematical Analysis. } \\
\text { Research in the didactics of Mathematics. }\end{array}$ \\
\hline
\end{tabular}

\section{Validation}

The system of activities that was originally structured in three questionnaires referred to exercises related to the three notions (TF, LD, and DPS); situations that look for the identification of the possibility to make the three developments (TF, LD, and DPS); then asked for examples in which some development (TF, LD, and DPS) is feasible and their explanations; and finally, asked for the development with more weight among them from the mathematical notion.

Experts A, D, and E established that, under the objective set out in this research and taking into account the academic background of the student to whom the activity is directed, it was necessary to consider activities that recognized if the students knew how to make the three developments in different situations, in the same situation and to identify their arguments about their differences.

Experts B and C suggested to clarify the reason for the interest in activities related to the development of series, to refine the general justification of research. Finally, on the proposed activities, they suggested to narrow down and explore the knowledge on series expansions (TF, LD, and DPS) and their differences.

Considering the first-hand validation information, it was decided to maintain three questionnaires, with step-by-step activities on the topics of interest.

\section{Questionnaires}

The three diagnostic forms were applied to a population of 67 students who were preparing to become university-level mathematics teachers at the Autonomous University of Guerrero Bachelor of Mathematics 
Degree. An academic background, the population of teachers training had studied the subjects of Differential and Integral Calculus (I, II, III and IV) in pre-college and Mathematical Analysis in this level of study.

\section{First questionnaire}

In this questionnaire, the students were asked to identify if the function $f(x)=e^{x}$ admits the three expansions, Taylor's Formula, limited development, and development of power series, in a neighborhood of 0; they should also justify their answers. This activity lasted 30 minutes.

\begin{tabular}{|c|c|c|c|}
\hline \multicolumn{4}{|c|}{ Activity. In the formula $e^{x}=1+x+\frac{x^{2}}{2 !}+\frac{x^{3}}{3 !}+\cdots$ the ellipsis “...” can be replaced by: } \\
\hline a) $\quad x^{3} \varepsilon(x)$ where $\lim _{x \rightarrow 0} \varepsilon(x)=0$ & YES & NO & I DON'T KNOW \\
\hline \multicolumn{4}{|l|}{ Justification: } \\
\hline b) $\quad x^{4} \beta(x)$ where $\lim _{x \rightarrow 0} \beta(x)=0$ & YES & NO & I DON'T KNOW \\
\hline \multicolumn{4}{|l|}{ Justification } \\
\hline c) $\frac{e^{c}}{4 !} x^{4}$ where $c$ is a real number such that $|c|<|x|$ & YES & NO & I DON'T KNOW \\
\hline \multicolumn{4}{|l|}{ Justification: } \\
\hline d) $\quad \sum_{n=4}^{+\infty} \frac{x^{n}}{n !}$ & YES & NO & I DON'T KNOW \\
\hline
\end{tabular}

\section{Second questionnaire}

An exercise that could be solved in two ways was proposed here. The first way involves Taylor's development and is a more immediate form to solve the problem; in fact, the given function is an odd function, and therefore it is enough to calculate $f(0)$ and $f^{\prime}(0)$. The second way is longer and involves using the classic theorems (addition, subtraction, multiplication, and so on, of the LD's) to find the solution.

The goal of this activity is to identify the first reactions of the students in an LD problem: Do they analyze the function to exploit their properties, or is their approach mechanical?

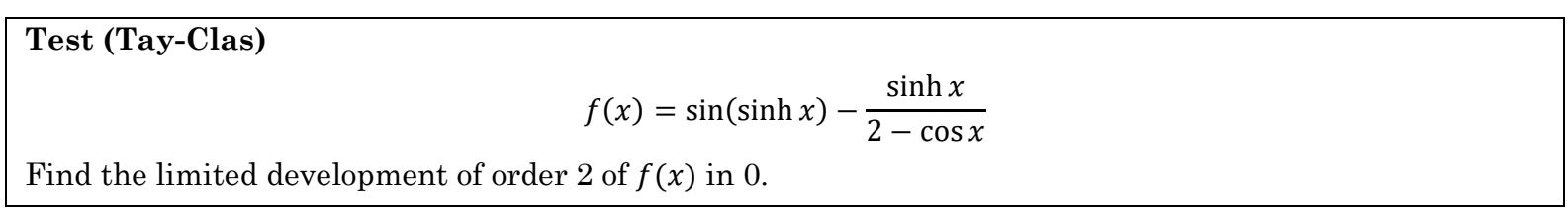

\section{Third questionnaire}

The only modification from the previous questionnaire made here was the argument of the sine function. In these conditions, if $\mathrm{x}$ tends to $0, \sin (\cosh x)$ tends to $\sin (1)$. In this case, if the composition theorem (which is less usual in this form) is to be applied, then it is necessary to find a development of power $\operatorname{series}$ of $\cosh x$ in 0 , and a development of power series of $\sin x$ in 1.

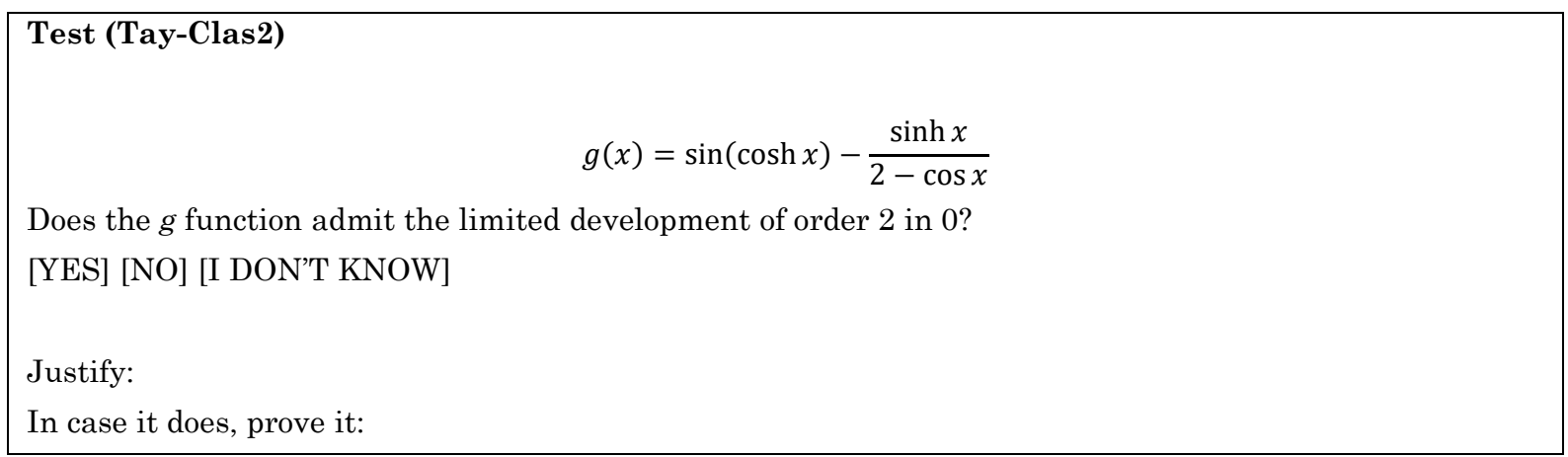




\section{ANALYSIS AND RESULTS}

\section{First Questionnaire}

Table 3 shows the results of the first questionnaire.

Table 3. Summary of the answers to the first questionnaire

\begin{tabular}{|c|c|c|c|c|c|c|c|c|c|c|c|}
\hline & & $\left(e^{x}\right.$ & & $\left(e^{x}\right.$ & & $\left(e^{x}\right.$ & & & & & \\
\hline & WI & $75 \%$ & & $21 \%$ & & $25 \%$ & & $28 \%$ & & $83 \%$ & \\
\hline & WJ & & $(51)$ & & (14) & & $(17)$ & & (19) & & $(55)$ \\
\hline Correct & IJ & $6 \%$ & & $6 \%$ & & $30 \%$ & & $6 \%$ & & $6 \%$ & \\
\hline answers & 10 & & (4) & & (4) & & $(20)$ & & (4) & & (4) \\
\hline & NJ & $8 \%$ & & $2 \%$ & & $18 \%$ & & $6 \%$ & & $4 \%$ & \\
\hline & NJ & & (5) & & (1) & & $(12)$ & & (4) & & (3) \\
\hline & & $6 \%$ & & $42 \%$ & & $11 \%$ & & $3 \%$ & & $4 \%$ & \\
\hline Incorrect & $J$ & & (4) & & (29) & & (7) & & (2) & & (3) \\
\hline answers & NJ & $2 \%$ & & $21 \%$ & & $4 \%$ & & $9 \%$ & & $0 \%$ & \\
\hline & Ne & & $(1)$ & & (14) & & (3) & & (6) & & $(0)$ \\
\hline I don't kn & & $3 \%$ & & $8 \%$ & & $12 \%$ & & $48 \%$ & & $3 \%$ & \\
\hline 1 GOH & & & $(2)$ & & (5) & & (8) & & (32) & & (2) \\
\hline Total nur & & $89 \%$ & & $28 \%$ & & $73 \%$ & & $40 \%$ & & $93 \%$ & \\
\hline correct ar & & & $(60)$ & & (19) & & $(49)$ & & $(27)$ & & $(62)$ \\
\hline Total nur & of & $8 \%$ & & $64 \%$ & & $15 \%$ & & $12 \%$ & & $4 \%$ & \\
\hline incorrect & jers & & (5) & & (43) & & (10) & & (8) & & (3) \\
\hline
\end{tabular}

\section{Details for the reading:}

1. Questions a), b), c), d) and e) have been called ( $\left.e^{x} x^{3} \varepsilon(x)\right),\left(e^{x} x^{2} \alpha(x)\right),\left(e^{x} x^{4} \beta(x)\right),\left(e^{x}\right.$ Tay) and $\left(e^{x} D P S\right)$, respectively.

2. The number in parentheses in each cell is the total number of students that answered the specific activity.

3. The responses of the students were first classified considering whether they were correct or incorrect, and then considering if their answer is Well Justified (WJ), Incorrectly justified (IJ) or Not Justified (NJ) (in the case their answers were correct) or Justified (J) or Not Justified(NJ) (in the case their answers were incorrect).

4. Let us clarify what we understand as "a correct and well-justified answer." For the question $\left(e^{x} x^{3} \varepsilon(x)\right)$, the meaning of "well justified" is to mention a definition or a precise theorem. For example, we have

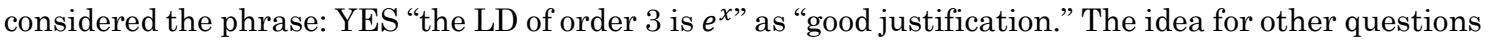
is similar. We can quote some examples of well-justified answers to the question $\left(e^{x}\right.$ Tay): YES "Taylor

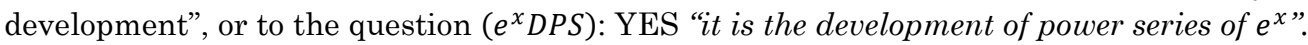

Table 3 shows that the question with more well-justified correct answers is $\left(e^{x} x^{3} \varepsilon(x)\right)$, (83\%). Furthermore, in questions $\left(e^{x} x^{3} \varepsilon(x)\right),\left(e^{x} x^{2} \alpha(x)\right)$ and $\left(e^{x} x^{4} \beta(x)\right)$, the justification of many students is based on the following arguments:

Example of a justification for the answer YES to the question $\left(e^{x} x^{3} \varepsilon(x)\right)$

$$
\begin{gathered}
" e^{x}=1+x+\frac{x^{2}}{2 !}+\frac{x^{3}}{3 !}+\frac{x^{4}}{4 !}+\frac{x^{5}}{5 !}+\cdots \\
e^{x}=1+x+\frac{x^{2}}{2 !}+\frac{x^{3}}{3 !}+3\left(\frac{x}{4 !}+\frac{x^{2}}{5 !}+\cdots\right)
\end{gathered}
$$

If we take $\varepsilon(x)=\frac{x}{4 !}+\frac{x^{2}}{5 !}+\cdots$,

then $\lim _{x \rightarrow 0} \varepsilon(x)=0$ os verified, and therefore the formula is correct".

Example of a justification for the answer NO to the question $\left(e^{x} x^{4} \beta(x)\right)$,

$$
\begin{gathered}
e^{x}=1+x+\frac{x^{2}}{2 !}+\frac{x^{3}}{3 !}+x^{4}\left(\frac{1}{4 !}+\frac{x}{5 !}+\cdots\right) \\
\beta(x)=\frac{1}{4 !}+\frac{x}{5 !}+\cdots, \text { therefore } \lim _{x \rightarrow 0} \beta(x) \neq 0
\end{gathered}
$$


The baseline of this argumentation is the idea of DPS, even when the questions refer the LD's.

The next quest was the observation of the proportion of well-justified answers to the questions that required the idea of DPS in their justifications. This argumentation is proof that $\mathrm{f}$ can be represented as a Limited Development of order 3 in 0 in question $\left(e^{x} x^{3} \varepsilon(x)\right)$, and that the existence of a limited development of order 3 does not imply the existence of a limited representation of order 4 in question $\left(e^{x} x^{4} \beta(x)\right)$. Table 4 shows the number of students whose argumentations were of this type.

Table 4. Responses of the teachers that have the idea of using DPS

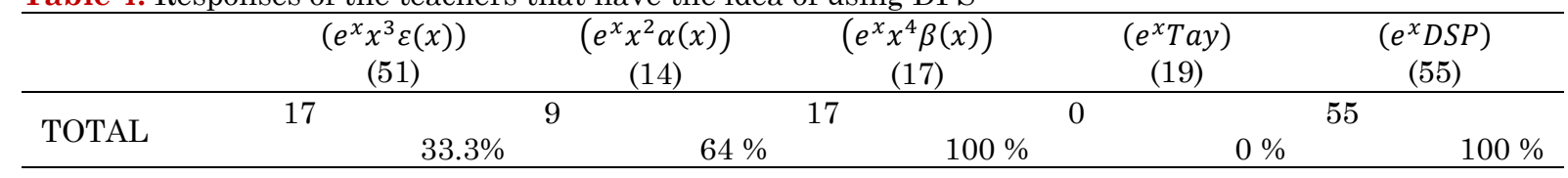

This percentage has been estimated considering the total number of well-justified answers in each question. The 34 students justified their answer by referring "LD of order 3 " in the first question. The other five students gave the following argument to justify their answer to the second question:

$$
\begin{gathered}
\text { YES “ } x^{3} \varepsilon(x)=x^{2} x \varepsilon(x)=x^{2} \alpha(x) \\
\text { with } \alpha(x)=x \varepsilon(x) \\
\text { If } \lim _{x \rightarrow 0} \varepsilon(x)=0 \text {, then } \lim _{x \rightarrow 0} \alpha(x)=0 "
\end{gathered}
$$

This means that they used the answer to the previous question to give their arguments.

From the last two rows in Table 3 (total number of correct and incorrect answers, respectively), it can be observed that the most successful question is $\left(e^{x} x^{3} \varepsilon(x)\right)$, which is connected to LD. However, taking into account the results in Table 4, it can be seen that the idea of DPS has played a very important role in the justifications of the correct answers to this question. Furthermore, a review of the correct answers, but incorrectly justified and the correct and well-justified answers to the questions $\left(e^{x} x^{2} \alpha(x)\right)$ and $\left(e^{x} x^{4} \beta(x)\right)$ could lead to a thorough analysis of the questions connected with LD. This information is summarized in Table 3.

Table 5. Correct answers incorrectly justified and incorrect answers with justification

\begin{tabular}{lcccc}
\hline & $\left(e^{x} x^{2} \varepsilon(x)\right)$ & & $\left(e^{x} x^{4} \beta(x)\right)$ \\
\hline Correct answers IJ & 4 & {$[Y E S]$} & 20 & {$[$ [NO] } \\
\hline $\begin{array}{l}\text { Incorrect answers with } \\
\text { justification. }\end{array}$ & & 29 & [YES] & \\
\hline
\end{tabular}

The first row in each cell contains the correct answers to the questions on the right, and the second row contains the incorrect answers to the questions on the left. The most significant answers are the justifications for [NO] to $\left(e^{x} x^{4} \beta(x)\right)$ (correct answer incorrectly justified,), and for [NO] to $\left(e^{x} x^{2} \alpha(x)\right)$ (Correct answer with Justification,). The arguments given by the students in both cases consider "the order of the LD does not agree with the degree of the regular part." Some examples are shown here:

[NO] "We went farther than order 2" (example of the justification of [NO] in $\left(e^{x} x^{2} \alpha(x)\right)$ )

[NO] "We stopped in the LD of order 3, $\frac{x^{4}}{4 !}$ would be missing" (example of the justification of [NO] in $\left.\left(e^{x} x^{4} \beta(x)\right)\right)$

A possible interpretation is that these students focus their attention only on the formal expression because this type of notation is always presented

$$
f(x)=a_{0}+a_{1} x+a_{2} x^{2} \ldots+a_{n} x^{n}+x^{n} \varepsilon(x),
$$

without thinking about the nature of the function $\varepsilon(x)$.

Table 5 shows that the proportion of justifications for [NO] in $\left(e^{x} x^{2} \alpha(x)\right)$ is similar to the proportion of justifications for [NO] in $\left(e^{x} x^{4} \beta(x)\right)$. This numbers leads us to think that, even when the percentage of success of question $\left(e^{x} x^{3} \varepsilon(x)\right)$ is high, it cannot be deduced that the LD notion is the one with the least difficulty. There is another comment related to the topic of comprehension of the expressions $x^{n} \varepsilon(x)$. Most of the justifications for [YES] (in this table), for both $\left(e^{x} x^{2} \alpha(x)\right)$ and $\left(e^{x} x^{4} \beta(x)\right)$, used this type of justifications.

$$
\text { “ } \lim _{x \rightarrow 0} \alpha(x)=0 \Rightarrow \lim _{x \rightarrow 0} x^{2} \alpha(x)=0 \text { “ (Example of a justification for [YES] in }\left(e^{x} x^{2} \alpha(x)\right) \text { ) }
$$




$$
\text { “ } \lim _{x \rightarrow 0} \beta(x)=0 \Rightarrow \lim _{x \rightarrow 0} x^{4} \beta(x)=0 " \text { (Example of a justification for [YES] in }\left(e^{x} x^{4} \beta(x)\right) \text { ). }
$$

There is not much information about the ideas of the students regarding the question related to the Formula of Taylor ( $e^{x}$ Tay), because few students justified their answers to this question. Most of the students that checked the cell [I DON'T KNOW] did not justify their answer, or wrote something like the following commentaries as justifications:

"I had problems remembering the formula; I hesitated between $\frac{e^{c}}{3 !} x^{3}$ and $\frac{e^{c}}{4 !} x^{4 "}$

"One of the formulas of Taylor? The integral remainder?"

Three students are in this situation.

Other observations that can be presented before we express the conclusions to this questionnaire are: (1) In the questions of LD (the first three), few students mentioned that the LD showed was in 0. (2) Only three students mentioned the order of the development presented in the question related to the formula of Taylor. By way of conclusion, we will confine ourselves to make some hypotheses based on the results. Which is the notion preferred by the students when a function admits the LD, TF, and DPS?

The first fact to point out is that the students seem to prefer the DPS notion for the problems of this nature. Thus, most of the students correctly answered and justified the question ( $\left.e^{x} D P S\right)$ related to the development of the power series of $e^{x}$. Furthermore, there is a significant proportion of students that used the argument of DPS to justify their answer even when the questions $\left(e^{x} x^{3} \varepsilon(x)\right),\left(e^{x} x^{2} \alpha(x)\right)$, and $\left(e^{x} x^{4} \beta(x)\right)$ made a limpid reference to the $\mathrm{LD}$.

Another reason that strengthens this conjecture is the fact that some students made the following type of observation at the end of their written answers, after finishing the questionnaire:

"The development in power series is valid on the radius of convergence, $x^{3} \varepsilon(x)$ is less accurate."

"This formula is only valid for an LD in a neighborhood of 0 ; it is not true for every real number $x$."

"For me, the ellipsis "..." represents what comes next in the formula

$$
\frac{x^{4}}{4 !}+\frac{x^{5}}{5 !}+\sum_{n=0}^{+\infty} \frac{x^{n}}{n !} "
$$

From our point of view, these students consider that the notion of DPS is the one with the "best properties." It is known that DPS is not a generalization of the other notions, but can it be considered as a unifying concept for these ideas? We will be cautious to answer this question and consider this study only as exploratory, but it will be very interesting to do other studies in the same direction immediately to reach definite conclusions.

However, we can comment that historically the notion of DPS has played a very important role in the development of the notions of convergence, uniform convergence, and the process of construction of the fundaments of the analysis in the nineteenth century. In this sense, we can consult the work of Duarte (1993) and take into account the consideration of Bottazzini (1986).

"There are two themes that cut across the history of mathematical analysis in the nineteenth century and form a kind of guiding line. One is the theory of series, particularly Fourier and power series; the other is the theory of real and complex integration" (p. 5)

\section{Second Questionnaire}

Table 6 shows the answers to this questionnaire.

Table 6. Summary of the answers to the second questionnaire

\begin{tabular}{cccc}
\hline & & Total & Percentage \\
\cline { 2 - 4 } Exact solution & Taylor & 6 & $9 \%$ \\
\cline { 2 - 4 } & Classic Theorems & 21 & $31 \%$ \\
\hline \multicolumn{2}{c}{ Total number of correct answers } & 27 & $40 \%$ \\
\cline { 2 - 4 } False solution & Taylor & 2 & $3 \%$ \\
\cline { 2 - 4 } & Classic Theorems & 33 & $49 \%$ \\
\hline \multicolumn{2}{c}{ Not answered } & 3 & $5 \%$ \\
\hline \multicolumn{2}{c}{ Miscellaneous } & 2 & $3 \%$ \\
\hline
\end{tabular}




\section{Details for reading the data}

1. Taylor (Exact solution or false solution). This case refers to the fact that students started solving the exercise using Taylor's formula.

In this rubric, the following was identified:

- Among the students that had this idea (exact solution or not), only three identified that function $\mathrm{f}$ is odd and that it was only about finding the first derivative in 0.

- In the rubric "False Solution-Taylor,"

- Two students were placed in this rubric: one did not finish the exercise, and the other wrote Taylor's formula at the beginning and then wrote the result

" $f(x)=x^{3} \varepsilon(x)$ ",

without writing the operations that would allow for the identification of the source of the mistake.

2. Not answered: It is about the students that leave the charts empty.

3. Miscellaneous: This final category groups the charts that contain written lines with no sense.

4. Classic theorems: (Exact or false solution): It is about the student that solved the problems using the Classic theorems.

As we stated previously, there are at least two routes for dealing with the fraction

$$
\frac{\sinh x}{2-\cos x}=\frac{x+x^{2} \varepsilon_{1}(x)}{1+\frac{x^{2}}{2 !}+x^{2} \varepsilon_{4}(x)}
$$

when the Classic Theorems are used to solve problems: Applying the theorem of the quotient of two Limited Developments, or Applying the theorem of the compound function, and then, the multiplication of two Limited Developments.

Table 7 shows how many of the students used each of these routes when using the Classic Theorems to solve the problem.

Table 7. Use of different routes

\begin{tabular}{ccc}
\hline Quotient & Compound function & Fraction \\
\hline 26 & 7 & 21 \\
\hline
\end{tabular}

The rubric "Fraction" regroups the students who stopped their processes of the solution as soon as they reach this part. Seventeen of these students concluded that

$$
\begin{aligned}
& \frac{\sinh x}{2-\cos x}=\frac{x}{1+\frac{x^{2}}{2 !}}+x^{2} \varepsilon(x) \\
& \frac{\sinh x}{2-\cos x}=\frac{2 x}{2+x^{2}}+x^{2} \varepsilon(x),
\end{aligned}
$$

this means that they did not do the quotient, but that this is the expression of the LD of order 2 of the fraction in 0 . From the results in Table 6, it can be deduced that the students would rather apply the "method" generated by the Classic Theorems. This could happen because it is an algorithmic method, and as said in the introduction, the students prefer using an algorithm to obtain a result quickly compared to the time expected using theoretical reasoning. However, the percentage of the "Exact solution" with this method is weaker than the percentage of the "False Solutions."

It is also necessary to observe that, among the students who obtained a false solution based on the Classic Theorems, there are four that did not write the correct expression of the remainders and two more that have only worked with the regular parts without writing the remainders from the beginning. Another student used the ellipsis “..." instead of the remainders in all the expressions.

\section{Third Questionnaire (First Part)}

Let us analyze the results of the question first, and then the analysis of the results of the exercise.

The answer to the question is [YES]. Table 8 shows the results. 
Table 8. Summary of the responses to the third questionnaire

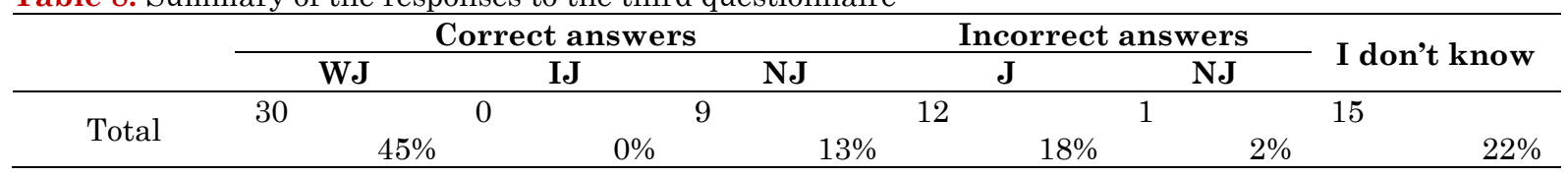

The correct answers in the first questionnaire have been classified as "Well Justified (WJ)," "Incorrectly Justified" (IJ) or "Not Justified (NJ)." Similarly, the incorrect answers could be classified as "Justified" (J), or "Not Justified" (NJ). Let us clarify what we understand as "Well-justified correct Answer": These answers are of two types. The first type groups justifications of the type:

[YES] "because $f$ is of class $C^{\infty}$.

Most of the answers justified correctly are of this type.

The second type, groups justifications of the type:

[Yes] "in 0, the value of $\cosh x$ is 1; it must be necessary to find an LD of $\sin$ in 1 .

Only five students gave justifications of this nature.

About the incorrect answers, the arguments of most of the students that made justifications are:

[NO] " $\cosh x \geq 1$. Therefore $\sin (\cosh x)$ has not a development in 0 ."

[NO] "because $\cosh x \approx 1$, and $\sin 1$ does not have an $L D . "$

[NO] " $x \mapsto \cosh x$ does not admit an LD in 0 (of any order). An LD of $g$ is needed; therefore, an LD of $x \mapsto$ $\sin (\cosh x)$ (a compound function) is needed. So, it is necessary that $x \mapsto \sin x$ and $x \mapsto \cosh x$ to have an LD in 0 ”.

This question was proposed in order to study the reactions of the students in the presence of an LD problem where the usual theorem of composition does not apply. Do they think of Taylor's formula? It is necessary to point out that, about the rubric [I DON'T KNOW], five of the fifteen students that were classified in this way did not cross out any checkbox. Another thing to point out related to this rubric is: One student who crossed out the checkbox [I DON'T KNOW] justified his answer in the following way:

[I DON'T KNOW]: “ $\cosh 0=1$ ”.

It is identified that this student did not know what action to take in this situation; this means, he perceived the problem but did not know how to deal with it.

Third questionnaire (second part). Table 9 summarizes the results of the exercise.

Table 9. Summary of the responses to the second part of the third questionnaire

\begin{tabular}{cccc}
\hline & & TOTAL & PERCENTAGE \\
\hline \multirow{2}{*}{ Exact Solution } & Taylor & 3 & $4.5 \%$ \\
\cline { 2 - 4 } & LD of the compound function & 0 & $0 \%$ \\
\hline \multirow{2}{*}{ Total number of correct answers } & Taylor & 3 & $4.5 \%$ \\
\hline \multirow{2}{*}{ Solution } & 6 & $9 \%$ \\
\cline { 2 - 4 } & LD of the compound function & 18 & $27.5 \%$ \\
\hline
\end{tabular}

Observation: No student answered the exercise using the composition theorem. This is not surprising because this theorem is rarely taught in this way.

The rubric "Not answered" is the most common answer. The 28 students that were classified in the rubrics "Incorrect answer" and "I DON'T KNOW" in Table 6 are included here; this is, most of the students that did not solve the exercises, answered that the given function did not have development. The majority of the other students answered [YES] without justifications. Only three students could reach a correct answer, and they all used Taylor's formula, and have presented that $g$ is a function of class $C^{\infty}$ as their justification. This is enough to apply Taylor's formula in this particular case, while the use of the Classic Theorems needed to have in mind that $\sin x$ must be developed in 1 . No student used this route.

\section{SOME COMPARISONS}

Let us compare the results of tests (Tay-Clas) and (Tay-Clas2). 
Table 10. Comparisons

\begin{tabular}{ccc}
\hline & (Tay-Clas) & (Tay-Clas2) \\
\hline Taylor & 8 & 9 \\
\hline Classic Theorems & 54 & 18 \\
\hline
\end{tabular}

More students had the idea of using the Classic Theorems to solve the exercise in both cases. However, the number of these students decreased in the test (Tay-Clas2). We believe that this happens because of the question posed before the exercise. This question seems to create a kind of "state of alert," and many students could not identify what it was about and chose not to do the exercise.

We can go further in the analysis of the "Classic Theorems" rubric of the last two questionnaires by analyzing the types of mistakes that the students with incorrect answers committed. We classify these mistakes into three types:

Miscalculations: The students who make mistakes when performing operations, or that, during the development of their work, changed the sign of a number, making false all subsequent procedures are placed in this rubric.

Method errors: The students who incorrectly applied any of the so-called classic theorems are classified in this rubric. For example, this is part of an answer to the test of Tay-Clas:

“...therefore

$$
\begin{gathered}
f(x)=x-\frac{x}{1+\frac{x^{2}}{2 !}+x^{2} \varepsilon(x)}+x^{2} \varepsilon^{\prime}(x) \\
=x-x\left(1-\frac{x^{2}}{2 !}\right)+x^{2} \varepsilon^{\prime \prime}(\mathrm{x}) \\
=\frac{x^{3}}{2 !}+x^{3} \varepsilon^{\prime \prime}(x) \\
\frac{1}{1+u}=(1+u)^{-1}=1-u+u^{2}+u^{2} \varepsilon(u)^{\prime \prime}
\end{gathered}
$$

This student did not correctly apply the theorem of a compound function to the fraction

$$
\frac{\sinh x}{2-\cos x}
$$

Table 7 shows other examples of mistakes in this rubric.

The mistakes of the test Tay-Clas 2 are of the same nature, and besides, all students but one developed the function $\sin x$ in 0 and not in 1.

Order: This rubric groups the students that apply the method correctly and without miscalculations, but get false results because, in the beginning, they wrote LD of the functions as:

$$
\begin{gathered}
" \sinh x=x+\frac{x^{3}}{3 !}+\frac{x^{5}}{5 !}+x^{5} \varepsilon(x) \\
\sin x=x-\frac{x^{3}}{3 !}+\frac{x^{5}}{5 !}+x^{5} \varepsilon(x) \\
\cosh x=1-\frac{x^{2}}{2 !}+\frac{x^{4}}{4 !}+x^{4} \varepsilon(x) "
\end{gathered}
$$

This is, they did not take into account the fact that the order requested was 2 . Some of them get even farther (for example, order 7); others did not write the expressions of the remainder, and three students wrote the following procedure:

$$
\begin{aligned}
& \sin x=x-\frac{x^{3}}{3 !}+\cdots \\
& \cosh x=1-\frac{x^{2}}{2 !}+\cdots
\end{aligned}
$$

$\mathrm{LD}$ of order 2 of $\sin (\sinh x)=x$; 


$$
\begin{gathered}
\text { LD of order } 2 \text { of } \frac{\sinh x}{2-\cos x}=\frac{x+\frac{x^{3}}{3 !}+\frac{x^{5}}{5 !} \ldots}{1+\frac{x^{2}}{2 !}-\frac{x^{4}}{4 !} \ldots}= \\
=x \frac{1+\frac{x^{2}}{3 !}+\frac{x^{4}}{5 !} \ldots}{1+\frac{x^{2}}{2 !}-\frac{x^{4}}{4 !} \ldots}
\end{gathered}
$$

Table 11 summarizes the results of this analysis.

Table 11. Summary of errors

\begin{tabular}{cccc} 
& Miscalculations & Method errors & Order \\
\hline (Tay-Clas) (33) & 7 & 17 & 9 \\
\hline (Tay-Clas2) (18) & 1 & 17 & 0 \\
\hline
\end{tabular}

Another observation for these two questionnaires is the fact related to the condition

$$
\lim _{x \rightarrow 0} \varepsilon(x)=0 .
$$

For the test Tay-Clas, from the 54 students who used the Classic Theorems, only 22 wrote the condition, 3 used the notation " $o(x)$ ", and the rest did not write anything in this sense. For the test Tay-Clas 2, 7 (of the total of 18) wrote the condition, 1 used the notation " $o(x)$ ", and the others did not write anything.

\section{CONCLUSIONS}

The results of the analysis of the first questionnaire highlight the idea that the Development of Power Series (DPS) played an important role in the justifications of the remaining questions. We can establish that of the three notions, the one that remains more often in the conception of the students is that of DPS. It is also concluded that the meaning of the ellipsis "..." is the most "concrete" for the students; the others present difficulties during their application or even confusion.

In the last two questionnaires, the analysis of the two exercises favored the identification that students prefer to apply the recipes generated by the Classic Theorems while solving the exercises instead of using Taylor's formula. However, most of them apply the method in a mechanical way, without reflecting on the conditions and properties of the functions to be developed, nor on the conditions that must be satisfied for the application of the Classic Theorems.

It was found that the students are used to manipulating only the first terms of the expressions and forget the sense of the complementary terms when analyzing the responses of each questionnaire.

In the first instance, the theoretical and methodological background motivated the conceptual review of the mathematical content of the study; subsequently, it favored the elaboration of the questionnaires and their serialization, considering the scope of the objective.

This work obtains theoretical and pedagogical elements that contribute to the project in process: methodological proposal for the treatment of the development of functions in power series for teaching at the university level.

\section{Disclosure statement}

No potential conflict of interest was reported by the authors.

\section{Notes on contributors}

Edgardo Locia Espinoza - Autonomous University of Guerrero, Mexico.

Armando Morales Carballo - Autonomous University of Guerrero, Mexico.

Héctor Merino Cruz - Autonomous University of Guerrero, Mexico. 


\section{REFERENCES}

Abarca, N. (2007). La enseñanza del cálculo diferencial e integral mediante la resolución de problemas, una propuesta motivadora. Revista Tecnociencia Universitaria Bolivia, 5(5), 14-20. https://doi.org/10.15359/ru.32-2.3

Antibi, A. (1988). Etude sur l'enseignement de méthodes de démonstration. Enseignement de la notion de limite: reflexions, propositions (State thesis). Universidad Paul Sabatier.

Ball, D., Thames, M., \& Phelps, G. (2008). Content knowledge for teaching: What makes its special? Journal of Teacher Education, 59(5), 389-407. https://doi.org/10.1177/0022487108324554

Bottazzini, U. (1986). The higher Calculus: a history of real and complex analysis, from Euler to Weierstrass. Springer Verlag. https://doi.org/10.1007/978-1-4612-4944-3

Carrillo, J., Aguilar, A., Contreras, L, Climent, N., Carmona, E., Escudero-Avila, D., ... Zakaryan, D. (2014). Un marco teórico para el conocimiento especializado del profesor de matemáticas. Huelva, Spain: Universidad de Huelva Publicaciones. https://doi.org/10.1080/14794802.2018.1479981

Duarte, A. (1993). Une introduction historique de la notion de convergence. Une étude sur la convergence simple de certains suites de fonctions. Memoria de D. E. A. Universidad Paul Sabatier.

Escudero-Ávila, D., Carrillo, J., Flores-Medrano, E., Climent, N., Contreras, L. C., \& Montes, M. (2015). El conocimiento especializado del profesor de matemáticas detectado en la resolución del problema de las cuerdas. Revista de Investigación en Didáctica de la Matemática, 10(1), 53-77. https://doi.org/10.30827/pna.v13i1.7944

Godino, J. (2009). Categorías de análisis de los conocimientos del profesor de matemáticas. Unión, Revista Iberoamericana de Educación Matemática, 20, 13-31. https://doi.org/10.1590/1980-4415v31n57a05

Huchecorne, B. (1988). Les contre-exemples en mathématiques. Paris, France: Ellipses.

Pichon, J. (1986). Calcul des limites. Paris, France: Ellipses.

Polya, G. (1958). Les mathématiques et le raisonnement plausible. Paris, France: Gauthier Villars.

Robert, A. (1982). L'acquisition de la notion de convergence de suites numériques dans l'enseignement supérieur (State Thesis). Universidad Paris III.

Shulman, L. (1986). Those who understand. Knowledge growth in teaching. Educational Researcher, 15(2), 414. https://doi.org/10.3102/0013189x015002004

Shulman, L. (1987). Knowledge and Teaching: Foundations of the New Reform. Harvard Educational Review, 57(1), 1-21. https://doi.org/10.17763/haer.57.1.j463w79r56455411 